\section{Faint X-ray sources in the core of the globular cluster M28}

\section{R. Danner ${ }^{\star} \dagger$, S. R. Kulkarni' ${ }^{\star}$, Y. Saito $\ddagger$ \& N. Kawai $\$$}

${ }^{*}$ Division of Physics, Mathematics and Astronomy 105-24, California Institute of Technology, Pasadena, California 91125, USA

$\dagger$ Max-Planck-Institute für extraterrestrische Physik, 85740 Garching, Germany ¥Institute of Space and Astronautical Science, 3-1-1 Yoshinodai, Sagamihara, Kanagawa 229, Japan

$\$$ The Institute of Physical and Chemical Research, 2-1 Hirosawa Wako, Saitama 351-01, Japan

Globular clusters, the most ancient stellar groups in our galaxy, are known to contain bright $\mathrm{X}$-ray sources, faint $\mathrm{X}$-ray sources and millisecond pulsars. The bright $X$-ray sources are neutron stars accreting matter from a companion star ${ }^{1}$, and the millisecond pulsars are believed to be descendants of these sources ${ }^{2}$. But the origin of the faint $X$-ray sources remains unclear. Here we report satellite-based X-ray observations of the globular cluster M28 which reveal two faint X-ray sources; an extended source slightly offset from the centre of the cluster, and a point source. The point source pulsates with the same period as a well-known ${ }^{3,4}$ 3-millisecond pulsar in M28. The nature of the extended source is more puzzling, however, and its spatial and spectral properties permit a range of plausible models. We argue that this source is either a collection of low-luminosity accreting neutron-star binaries or a synchrotron nebula powered by a recent outburst of energy from an unknown source. Sensitive optical and X-ray observations should be able to distinguish between these two possibilities.

The globular cluster M28 was observed with the High Resolution Imager (HRI) aboard the X-ray observatory satellite Rosat ${ }^{5}$ between 18 March and 21 April 1995. The on-source integration time was $41.7 \mathrm{ks}$. Our HRI image shows that the X-ray source RX J1824.5 - 2452 discussed earlier ${ }^{6,7}$ breaks up into separate sources (Fig. 1): a point source RX J1824.5 - 2452P and an extended source RX J1824.5 - 2452E. No other source is seen in the 1-degree field of view of the HRI. The count rate within an aperture of 7 arcsec centred on each source is $3.7 \times 10^{-3} \mathrm{~s}^{-1}$ for RX J1824.5 - 2452P and $7.7 \times 10^{-3} \mathrm{~s}^{-1}$ for RX J1824.5 - 2452E. The background count rate, normalized to the same aperture radius, was found to be $0.2 \times 10^{-3} \mathrm{~s}^{-1}$. The radius of the aperture is a compromise choice in view of the small separation, $9.3 \mathrm{arcsec}$, between the two sources. The sum of the two source count rate is well determined; the individual count rates depend on the details of the photometry. We conservatively estimate an uncertainty of $20 \%$ in the individual count rates.

M28 was also observed by the X-ray satellite $\mathrm{ASCA}^{8}$. The analysis of these data is reported elsewhere ${ }^{4}$. The angular resolution of ASCA compared to that of the HRI is poor, a few arcminutes, instead of a few arcseconds. Thus the two sources discussed above are seen as only one source in the ASCA data. However, ASCA has broad-band coverage $(1-10 \mathrm{keV})$ and is well suited for spectroscopy. Pulsations at the period of the 3-ms ratio pulsar B1821-24 (ref. 3) were detected in this data. We carried out a similar search for the HRI data and confirm the X-ray pulsations seen in the ASCA data (Fig. 1). By tagging each HRI photon detection with its corresponding phase bin, we created two images: one when the pulsar is 'on' and the other when it is 'off' (Fig. 1). These two images clearly demonstrate that RX J1824.5 - 2452P is indeed the pulsar.

The ASCA spectrum can likewise be separated into two parts ${ }^{4}$. The ASCA spectrum of the sum of the pulsed and unpulsed components is well described by a power-law spectrum and a neutral hydrogen column density, $N_{\mathrm{H}}=2.8 \times 10^{21} \mathrm{~cm}^{-2}$. This is consistent with the value extrapolated from the observed dispersion measure to the pulsar. As can be seen from Figs 1 and 2, the unpulsed signal is entirely due to RX J1824.5 - 2452E. Our ASCA spectrum of the non-pulsed signal may be well described by a power-law model with photon index $1.94 \pm 0.12$, or an optically thin thermal model with $k T=4.2_{-1.0}^{+1.6}$, or a thermal bremstrahlung model with $k T=6.0_{-1.0}^{+3.5}$, where $T$ is temperature. We used the program PIMMS (Portable Interactive Multi-mission Simulator), developed at the Goddard Space Flight Center, to convert the HRI photon rates to flux. We applied the ASCA spectral models to the pulsed and unpulsed signals and obtained the following unabsorbed fluxes in the ROSAT HRI energy range of $0.1-2.4 \mathrm{keV}$ :
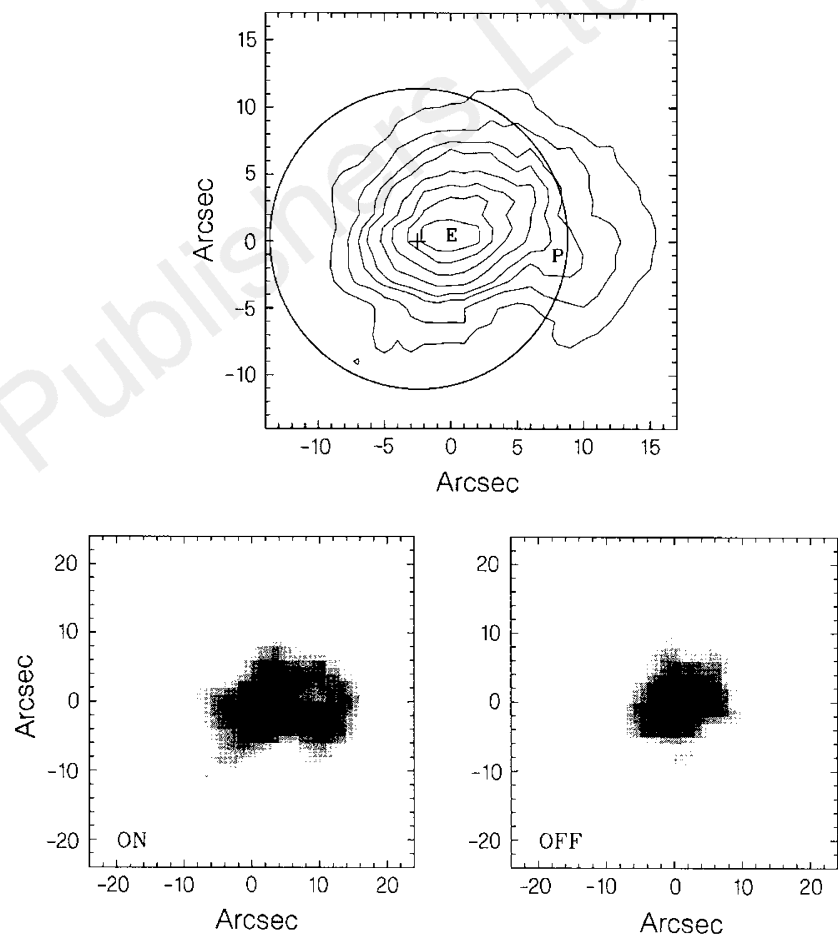

Figure 1 Rosat HRI image of the sources in M28. The HRI photon events were binned into an image with a pixel scale of 1 arcsec per pixel. The image was then convolved with a two-dimensional gaussian function with $\sigma=2$ pixels. The resulting spatial resolution of the image is quite close to the width of the pointspread function, 2.2 arcsec, derived from ground-based calibration and in-flight observations ${ }^{20}$. The orientation in all images is north up and east to the left. Top panel, contour plot created from 41.7-ks of integration time. The source in the core of M28 clearly shows a resolved structure. The positions of RXJ1824.5-2452P(P) and RXJ1824.5 - 2452E (E) are marked. The cross and the circle mark the optical centre and core radius of $\mathrm{M} 28$; these were obtained from refs 21,22 . For this plot the $\mathrm{X}$-ray image has been shifted so that the pulsed $X$-ray source $R X$ J1824.5-2452P coincides with the radio position of pulsar B1821-24. A horizontal bar indicates the image scale. The fact that HRI events are timetagged (to a precision of $60 \mu \mathrm{s}$ ) allows us to study pulsations from the 3-ms radio pulsar known to be in the core of this cluster. To this end we restricted the analysis to the first $29.3 \mathrm{ks}$ of data; these data were obtained over $70 \mathrm{~h}$. The radio barycentric period at the start of the Rosat observation (JD 2,449,795.0) was extrapolated from the radio ephemerides of Cognard et al. ${ }^{23}$ as $3.054,314,886,818 \mathrm{~ms}$. Over the $70 \mathrm{~h}$ of observing time, this period is expected to change by $<0.5 \mathrm{ps}$. The folded data were subject to $a Z^{2}$ test ${ }^{24}$; we found $Z_{4}^{2}=34.4$. Such a high value is found by chance at a probability level of $10^{-6}$. X-ray pulsations from the pulsar have clearly been detected. See Fig. 2 for the resulting pulse profile. The bottom panels compare the two sources during the on-phases and the off-phases of pulsar B1821 - 24. For the on-phase image, photons from phase 0.3-0.4 and phase 0.8-0.9 were combined. For off-phase image, photons from phase 0.0-0.2 were combined with photons from phase 0.5-0.7. Here it is clear that RXJ1824.5 - 2452 consists of two independent sources. 
$S_{\mathrm{P}}=3.9 \times 10^{-13} \mathrm{erg} \mathrm{cm}^{-2} \mathrm{~s}^{-1}$ and $S_{\mathrm{E}}=1.1 \times 10^{-12} \mathrm{erg} \mathrm{cm}^{-2} \mathrm{~s}^{-1}$ (here and below the subscripts $\mathrm{P}$ and $\mathrm{E}$ stand for the pulsar and the extended source, respectively).

What is RX J1824.5 - 2452E? It is clearly extended, which renders unlikely the hypothesis that this is a background active galactic nucleus (AGN) or a foreground interloper (a star with an active corona). The extended emission could possibly be due to a galaxy or a cluster of galaxies. The expected total number of extragalactic sources is 3 per square degree for $S>10^{-13} \mathrm{erg} \mathrm{cm}^{-2} \mathrm{~s}^{-1}$ (ref. 9). According to ref. 10, most of these sources are due to AGN with the galaxies contributing $6 \%$ and clusters a mere $1 \%$. Galaxies dominate only at flux levels well below $10^{-13} \mathrm{erg} \mathrm{cm}^{-2} \mathrm{~s}^{-1}$. Thus, from a statistical point of view, it is unlikely that RX J1824.5-2452E is emission from a galaxy. The close location of RX J1824.5 - 2452E to the centre is suggestive of the source being a cluster. Accordingly, we now consider models in which RX J1824.5-2452P is an X-ray source within the globular cluster M28.

Adopting a distance to RX J1824.5 - 2452P of $5.1 \mathrm{kpc}$ (ref. 11), we derive isotropic luminosities (in the Rosat band) of $L_{\mathrm{P}}=1.1 \times 10^{33} \mathrm{erg} \mathrm{s}^{-1}$ and $L_{\mathrm{E}}=3.3 \times 10^{33} \mathrm{erg} \mathrm{s}^{-1}$. A plausible model for RX J1824.5-2452E under the assumption that this source is in M28 must account for the following three observations: (1) the source is extended, $\sim 0.26 \mathrm{pc}$ at the distance to M28; (2) the source is within the core radius but not located at the centre of M28; and (3) the X-ray spectrum of the source is hard. We now consider two classes of models for RX J1824.5 - 2452E: a nebular origin and a stellar origin.

In the first model, the extended emission is from a nebula. Krockenberger and Grindlay ${ }^{12}$ have noted diffuse X-ray emission in the globular cluster 47 Tucanae; not much is known about its spectral properties. These authors explain the lack of a corresponding radio nebula by suggesting that the $\mathrm{X}$-ray emission arises from Compton scattering of optical photons by a pool of energetic particles. The model does not explain how the energetic particles are confined and we consider this as a fatal limitation of the hypothesis. A more conventional explanation is that $\mathrm{RX}$
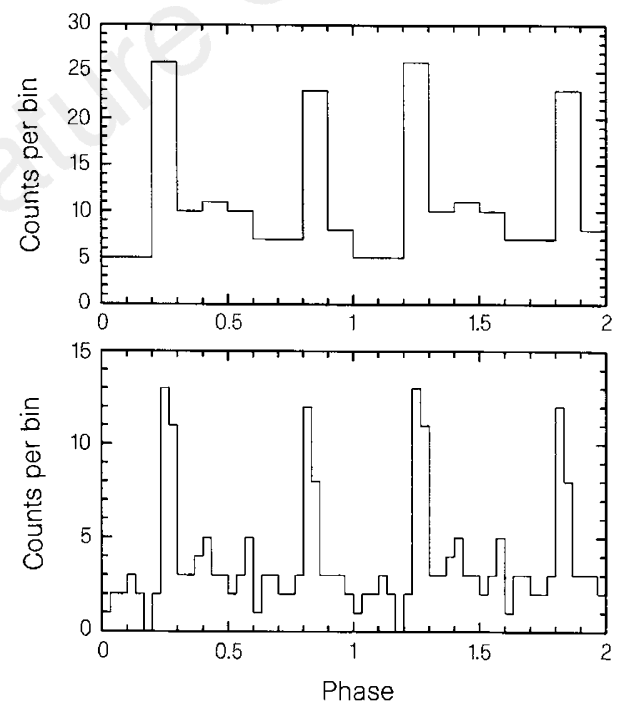

Figure 2 X-ray pulse profile of pulsar B1821-24 taken with the Rosat HRI. See Fig. 1 legend for details of the timing analysis. A total of 112 photons was detected within 7 arcsec of the radio position. In the top panel, the data are shown at a resolution of 10 phase bins; in the bottom panel, the same data are shown at a resolution of 30 phase bins. The profile shows two very narrow peaks. The calibration of the Rosat clock does not allow us to phase-reference, with the required degree of precision, the $\mathrm{X}$-ray light curve with the radio pulse profile. Therefore we choose an arbitrary zero phase point.
J1824.5 - 2452E is a synchrotron nebula similar to the Crab nebula. The X-ray spectrum of the Crab nebula is well described by a power law with a photon index of 2; This index becomes 1.3 below the so-called 'break frequency', $\nu_{\mathrm{B}}$. For RX J1824.5 - 2452E the spectral flux density at frequency $\nu_{\mathrm{B}}=3 \times 10^{17} \mathrm{~Hz}$ (corresponding to photons with energy of $1 \mathrm{keV}$ ) if $f_{\mathrm{X}}=0.4 \mu \mathrm{Jy}$. Thus the expected flux at a frequency $\nu$ (with $\nu<\nu_{\mathrm{B}}$ ) is $f_{\mathrm{B}}\left(\nu / \nu_{\mathrm{B}}\right)^{-0.3}$; here $f_{\mathrm{B}}=f_{\mathrm{X}}\left(\nu_{\mathrm{B}} / \nu_{\mathrm{X}}\right)^{-1}$ is the spectral flux at the break frequency. There is no radio counterpart to this nebula at $\nu=1.4 \mathrm{GHz}, f<1 \mathrm{mJy}$ (ref. 13). Thus $\nu_{\mathrm{B}}$ must be greater than $\sim \nu_{\mathrm{X}} / 20$. In contrast, $\nu_{\mathrm{B}}$ for the $\mathrm{Crab}$ and other pulsar powered nebulae is in the infrared or radio frequencies. Using the above value of $\nu_{\mathrm{B}}$ we derive the luminosity of the nebula across the entire electromagnetic spectrum. The standard synchrotron nebula model ${ }^{14}$ relates this to the energy in particles and nebular magnetic field strength. The total energy is related to the integrated luminosity of the nebula; using the above upper limit at $1.4 \mathrm{GHz}$, we obtain an upper limit to the integral luminosity of $8 \times 10^{33} \mathrm{erg} \mathrm{s}^{-1}$. The corresponding upper limit to the total energy in particles is $10^{45} \mathrm{erg}$ and the equipartition nebular magnetic field strength is below $87 \mu \mathrm{G}$. Energetic electrons gyrate in this magnetic field and lose energy. The lifetime of the electrons which produce the photons of energy $h \nu_{\mathrm{B}}$ is a rough measure of the age of the nebula; we find this to be greater than $200 \mathrm{yr}$. However, this age is much smaller than the $3 \times 10^{7}$ yr age of the $3-\mathrm{ms}$ pulsar ${ }^{15}$. This discrepancy is so large that we abandon the model in which the nebula is powered by the 3-ms pulsar. Preserving the synchrotron nebula model requires that, $\sim 200 \mathrm{yr}$ ago, there was an energetic event in the core of M28 with a total energy of $10^{45} \mathrm{erg}$.

In the stellar model RX J1824.5-2452E is due to a number of faint $X$-ray sources. The sources could be accreting binaries containing white dwarfs (cataclysmic variables, $\mathrm{CVs}$ ) or neutron stars (lowmass X-ray binaries (LMXBs) of low luminosity). HRI observations of one of the nearest globular clusters, NGC6397, reveal the presence of faint sources with $\mathrm{X}$-ray luminosity $L_{\mathrm{X}} \approx(2-10) \times$ $10^{31} \mathrm{erg} \mathrm{cm}^{-2} \mathrm{~s}^{-1}$ (refs 16, 17). Grindlay et al. ${ }^{18}$ have reported optical data and argue that these faint sources are magnetic CVs. A few tens of such CVs could then account for RX J1824.5 - 2452E. However, the X-ray spectra of nearby magnetic CVs are very hard with a characteristic temperature of $30 \mathrm{keV}$, whereas the characteristic temperature of RX J1824.5-2452E is $5 \mathrm{keV}$. This then leads us to consider that the faint X-ray sources are faint LMXBs, that is, accreting neutron-star systems. An example of such a system is Centaurus $\mathrm{X}-4$ which has $L_{\mathrm{X}} \approx 2 \times 10^{32} \mathrm{erg} \mathrm{s}^{-1}$. The ASCA spectrum of Cen X-4 can be explained by a combination of $0.3-\mathrm{keV}$ black body and a long hard-spectrum tail ${ }^{19}$. It is plausible that the blackbody component arises from the heated surface. Depending on the accretion history, it is possible to have the hard-spectrum tail dominate over the backbody component. The non-central location of RX J1824.5 - 2452E may seem to be a problem. However, we do not consider this to be the case for two reasons. First, the noncentral location could be due to chance fluctuations. Second, the displacement is small and, given the statistics of a small number of photons, it is entirely possible that the displacement from the centre is not statistically significant.

Thus we have developed two plausible models for RX J1824.5 - 2452E: a synchrotron nebula model powered by an energetic event that took place $\sim 200 \mathrm{yr}$ ago, or collective emission from a dozen faint LMXBs. The two possibilities are distinguishable. The nebula may be detectable at optical frequencies, $\nu_{\mathrm{O}} \approx 6 \times 10^{14} \mathrm{~Hz}$, with a surface brightness of $26 \mathrm{mag} \mathrm{arcsec}^{-2}$. The LMXB model would be favoured if any variation in the X-ray flux of RX J1824.5 - 2452E were to be detected; this is because most LMXBs are variable sources.

Note added in proof: J. E. Grindlay (personal communication) has suggested that the spectral properties of the population of faint sources discussed here are also consistent with a population of socalled DQ Her CVs. 
1. Lewin, W. H. G., van Paradijs, J. \& van den Heuvel, E. (eds) X-ray Binaries (Cambridge Univ. Press, 1995)

2. Alpar, M. A., Cheng, A. F., Ruderman, M. A. \& Shaham, J. A new class of radio pulsars. 300, 728-730 (1982)

3. Lyne, A. G. et al. The discovery of a millisecond pulsar in the globular cluster M28. Nature 328, 399401 (1987)

4. Saito, Y. et al. Detection of magnetospheric X-ray pulsations from the millisecond pulsar B1821-24. Astrophys. J. 477, L37-L40 (1997).

5. Trumper, J. ROSAT. Phys. Scripta T 7, 209-215 (1984)

6. Johnston, H. M., Verbunt, F. \& Hasinger, G. ROSAT PSPC observations of globular clusters. Astron Astrophys. 289, 763-774 (1994).

7. Danner, R., Kulkarni, S. R. \& Thorsett, S. E. ROSATobservations of six millisecond pulsars. Astrophys. J. 436, L153-L156 (1994)

8. Tanaka, Y., Inoue, H. \& Holt, S. S. The X-ray astronomy satellite ASCA. Publ. Astron. Soc. Jpn 46, L37L41 (1994).

9. Hasinger, G. et al. A deep X-ray survey in the Lockman hole and the soft X-ray $\log \mathrm{N}-\log \mathrm{S}$. Astron. Astrophys. 275, 1-5 (1993).

10. Almaini, O. et al. A deep ROSAT survey. 12. The X-ray-spectra of faint ROSAT sources. Mon. Not. $R$ Astron. Soc. 282, 295-303 (1996).

11. Rees, R. F. \& Cudworth, K. M. A new look at the globular cluster M28 Astron. J. 102, 152-158 (1991).

12. Krockenberger, M. \& Grindlay, J. E. Discovery of diffuse X-ray emission in 47-tucanae. Astrophys. J. 451, 200-209 (1995).

13. Kulkarni, S. R., Goss, W. M., Wolszczan, A. \& Middleditch, J. Deep radio synthesis images of globular clusters. Astrophys. J. 363, L5-L8 (1990).

14. Pacholczyk, A. G. Radio Astrophysics Ch. 7 (Freeman, San Francisco, 1970).

15. Foster, R. S., Backer, D. C., Taylor, J. H. \& Goss, W. M. Period derivative of the millisecond pulsar in the globular cluster M28. Astrophys. J. 326, L13-L15 (1988).

16. Verbunt, F., van Paradijs, J. \& Elson, R. X-ray sources in globular clusters. Mon. Not. R. Astron. Soc. 210, 899-914 (1984).

17. Hasinger, G., Johnston, H. M. \& Verbunt, F. Discovery of multiple X-ray sources in 47-Tucanae. Astron. Astrophys. 288, 466-471 (1994).

18. Grindlay, J. E. et al. Spectroscopic identification of probable cataclysmic variables in the globular cluster NGC-6397. Astrophys. J. 455, L47-L50 (1995).

19. Asai, K. et al. ASCA observations of soft X-ray transients in quiescence-X1608-52 and Cen X-4.Publ. Astron. Soc. Jpn 48, 257-263 (1996).

20. David, L. P., Harnden, F. R., Kearns, K. E. \& Zombeck, M. V. in The ROSAT High Resolution Imager Calibration Report 1-52 (SAO, Cambridge, MA, 1996).

21. Djorgovski, S. \& Meylan, G. in Structure and Dynamics of Globular Clusters (eds Djorgovski, S. G. \& Meylan, G.) 325-336 (Astron. Soc. Pacif., San Francisco, 1993).

22. Trager, S. C., Djorgovski, S. \& King, I. R. in Structure and Dynamics of Globular Clusters (eds Djorgovski, S. G. \& Meylan, G.) 347-355 (Astron. Soc. Pacif., San Francisco, 1993).

23. Cognard, I. et al. High precision timing observations of the millisecond pulsar PSR B1821-24 at Nancy. Astron. Astrophys. 311, 179-188 (1996).

24. Buccheri, R. \& De Jaeger, O. C. in Timing Neutron Stars (eds Ögelman, H. \& van den Heuvel, E. P. J.) 95-111 (Kluwer, Dordrecht, 1989).

Acknowledgements. We thank J. Grindlay and F. Verbunt for discussions. R.M.D. and S.R.K. thank NASA for support. This work was partially supported by the Scientific Research Fund of the Japanese Ministry of Education, Science, and Culture.

Correspondence should be addressed to S.R.K. (e-mail: srk@astro.caltech.edu)

\section{Turbulent drag reduction by passive mechanisms}

\section{Sirovich \& S. Karlsson}

Orlev Scientific/Ormat Industries, PO Box 68, Yavne, 70650 Israel, and The Division of Engineering, Brown University, Providence, Rhode Island 02912, USA

In many situations involving flows of high Reynolds number (where inertial forces dominate over viscous forces), such as aircraft flight and the pipeline transportation of fuels, turbulent drag is an important factor limiting performance. This has led to an extensive search for both active and passive methods for drag reduction $^{1}$. Here we report the results of a series of wind-tunnel experiments that demonstrate a passive means of effectively controlling turbulence in channel flow. Our approach involves the introduction of specified patterns of protrusions on the confining walls, which interact with the coherent, energy-bearing eddy structures in the wall region, and so influence the rate at which energy is dissipated in the turbulent flow. We show that relatively small changes in the arrangement of these protrusions can alter the response of the system from one of drag decrease to increased mixing (drag enhancement).

Experiments were carried out in a fully developed turbulent channel flow of half-height $h=2.815 \mathrm{~cm}$, width $75 \mathrm{~cm}$ and length $8.5 \mathrm{~m}$. (A description of the channel may be found elsewhere ${ }^{2}$.) the range of Reynolds numbers $(\mathrm{Re})$ of the channel, based on centre- line velocity, $U_{0}$, was $1.5 \times 10^{4}<\operatorname{Re}=U_{0} 2 h / \nu<4 \times 10^{4}$, where $\nu$ is the kinematical viscosity. The flow therefore falls into the realm of fully developed turbulence downstream of the entry region ${ }^{3}$. By convention, such turbulent flows are viewed as having a central core flow which is referred to as the outer region, in contrast with the layer adjacent to the wall, referred to as the inner region. In the former region the characteristic length scale is the channel halfheight $h$ which, with $U_{0}$, defines a 'turnover' timescale, $\tau_{0}=h / U_{0}$. Both $h$ and $\tau_{0}$ represent estimates of scales over which correlations are lost. Further, in the outer region viscosity plays an asymptotically small role and the mean velocity, $U(y)$, scales as $U_{0}-U(y)=$ $U_{0} f(y / h)$, where $y$ is the wall-normal distance. The inner velocity scale is the friction velocity, $u_{*}$, defined by the wall friction, $u_{*}^{2}=\nu[\partial U / \partial y]_{y=0}$. The inner length scale is then given by $l_{*}=\nu / u_{*}$, and the mean velocity scales as $U(y) / u_{*}=g\left(y / l_{*}\right)=g\left(y_{*}\right)$, referred to as the law of the wall. $u_{*}$ defines a second Reynolds number, $\operatorname{Re}_{*}=u_{*} h / \nu$, on which basis $750<\operatorname{Re}_{*}<2,000$ in our experiments.

Both $f(y / h)$ and $g\left(y_{*}\right)$ are regarded as being universal functions. Postulation of an overlap region ${ }^{4,5}$ leads to the 'log-law', $U / u_{*}=$ $\kappa^{-1} \ln y_{*}+B$ where $\kappa$, the von Karman constant, and $B$ are empirically determined. Evidence of the log-law, in an investigation of pipe flow over three decades of Re (ref. 6), indicates a logarithmic overlap region at high $\operatorname{Re}$ (ref. 7 ).

Several features of the flow show universality. In particular fluctuations in the direction of the flow $\left(u_{\mathrm{rms}}\right)$ and turbulence production $(-\overline{-u v} \mathrm{~d} U / \mathrm{d} y)$ peak at $y_{*} \approx 14$. Here $u$ and $v$ denote velocity fluctuations in the streamwise and wall-normal directions, respectively, and $\overline{u v}$, the Reynolds stress, is the temporal covariance. Of particular importance is the universal presence of 'streaks' in the wall region ${ }^{8,9}$, which refer to counter-rotating rolls, aligned in the stream direction, that confer on the flow a (statistically) quasiperiodicity in the transverse direction of wavelength $\lambda \approx 100 l_{*}$ (ref. 10). ('Transverse' here indicates the direction across the width of the channel.) Simulations indicate that these are tubular structures, of positive or negative vorticity with respect to the stream, which extend in the stream direction for as much as $1,000 l_{*}$ (ref. 11). Simple scaling arguments suggest that roll spacing should grow linearly with wall-normal distance, and this has been verified experimentally ${ }^{2}$. A variety of mechanistic theories have been suggested to explain the presence of streaks ${ }^{12-14}$, with no general agreement. More recently a statistical theory ${ }^{15}$ suggests that the rolls are the result of an inverse cascade ${ }^{16,17}$; that is, instead of the normal cascade of turbulence to smaller scales, this cascade takes the flow to larger scales. The spacing is then well estimated by balancing turbulence production and the rate of ejected energy from the wall region.

It has long been known that these structures undergo a rapid cycle of events culminating in their eruption from the wall region, known as 'bursts', which results in slow-moving wall fluid entering the outer region and fast-moving outer fluid entering the wall region; the latter are known as 'sweeps ${ }^{18-20}$. These relatively infrequent events account for a significant fraction of wall drag. One view of this phenomenon is that if coherent structures could be stabilized drag would be reduced. (Or from a more general perspective, stabilized coherent structures present a barrier to the cascade to small scales, and hence their maintained presence would impede the rate of energy loss.) Explanations of drag reduction by means of riblets use this viewpoint ${ }^{21}$. Riblets (wall grooves aligned with the stream, with transverse wavelength and height $\sim 15 l_{*}$ ), which in physical experiments can produce drag reductions of $\sim 6 \%$ (refs 21 , 22 ), and $\sim 4 \%$ in simulations ${ }^{23,24}$, are thought to restrain the movement of the rolls and therefore maintain their coherence. The methods of turbulence control presented here differ radically from this approach.

Analysis of several numerical simulations ${ }^{25,26}$ reveals that turbulent energy resides primarily in stream-independent (roll) modes. Further, this analysis also revealed the presence of propagating plane 